Vol. 1, No. 1 | January - June 2018

\title{
Design and Control of an Unmanned Ground Vehicle for Search and Rescue Missions
}

\author{
Kamran Shahani ${ }^{1}$, Hong Song ${ }^{1}$, Chaopeng Wu ${ }^{1}$, Syed Raza Mehdi ${ }^{1}$, Kazim \\ Raza $^{1}$, Noorudin Khaskheli ${ }^{2}$
}

\begin{abstract}
:
This paper focuses on the design and control of the unmanned ground vehicle for search and rescue missions. It is operated manually by using Xbee remote controller and arduino Uno is utilized to guide signals to UGV. This robot has innovations such as to move on rocky surfaces, a robotic arm is fixed on it which will perform pick and place tasks according to the inputs from the operator. The camera is attached for live vision feedback. The custom model designed was experimented with various situation scenarios to test its fidelity. This project is suitable for multiple purposes like monitoring and for military exploration missions.
\end{abstract}

Keywords: Xbee module; UGV; Arduino; Wireless Control; DC motor; Manipulator.

\section{Introduction}

An Unmanned Ground Vehicle (UGV) is a vehicle running in touch with the ground, transporting devices, without a human operator presence. The robot can move on the rough grounds and in order to perform various farm duties where the presence of human being inside the area/place is unsafe [1]. To evade trailing of human life, this is more suitable to use the robot for a related job. Mostly UGV's are useful for nowadays for the armed forces especially in the battlefield or war, now a day's these are also used in different industries for loading and unloading purpose, in nuclear power plants for filling and wasting purpose and in homes, these robots are used as servants [2]. The aim is to get the wireless live visual feedback of the accident cities, find the presence of enemy's positions, weapons, obstacles and much more information without any distressing they can get it control [3]. The UGV can work as rescue robot in rescue organization, rescue organization will quickly and securely receive data of the accident cities, a duty that is both tough and terrible.

A review of the development of unmanned ground vehicles in Japan from the1970's to 1990's is presented in [4].The Unmanned Ground Vehicle for fumigation purpose which designed by the National Space Research and development agency, Abuja Nigeria [5]. The vehicle consists of four wheels, uses Bluetooth as communication channel and can operated around 8 meters of radius.

${ }^{1}$ Ocean optics and automation lab, Institute of ocean technology, Ocean College, Zhejiang University Hangzhou 310058, China;

${ }^{2}$ Institute of Coastal engineering, Ocean College, Zhejiang University

Corresponding Email: 21634142@zju.edu.cn 


\section{Proposed System}

Figure 1 shows the entire proposed system block diagram where the Arduino Uno microcontroller saves the $\mathrm{C}$ code that commands the vehicle, the manipulator drive and the camera. The two direct current (DC) motors drive the vehicle forth, backward, left and right directions, the servo motor controls the movements of manipulator and camera. L298 driver module works as the direct current (DC) motors driver board and the Xbee module builds a wireless connection between the surface controller and the vehicle [6].

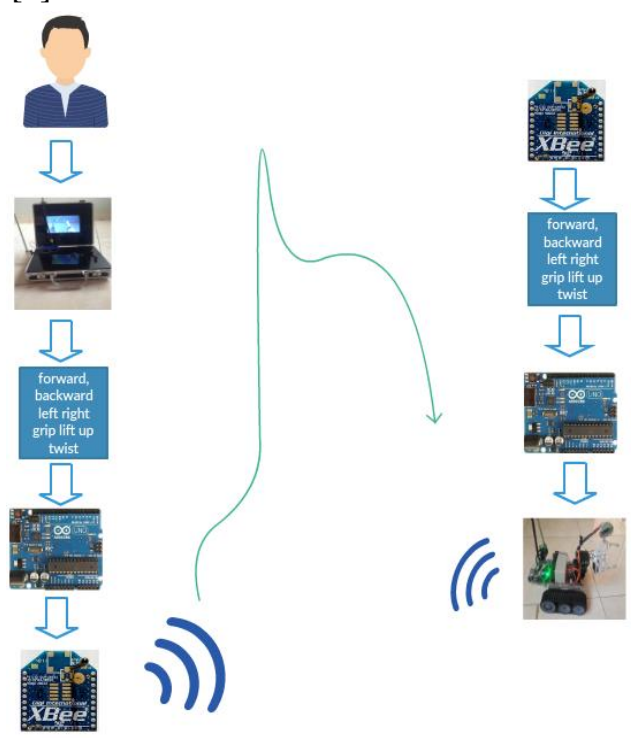

Fig. 1. Proposed System block diagram.

\subsection{Versa Chassis Body}

We have built essentially the frame of our vehicle from Versa (VEX) apparatus [7]. These components are made of a lightweight aluminum sheet. We use this because it saves our lot of time all parts are available we have to just assemble and build the body of a robot. Other parts are also available such as a shaft, gears, bearings gripper and many more. On aluminum sheets there are already holes and squares available; the size of these holes is about $0.8 \mathrm{~cm}$. For assembling the robot we do not need any welding machine for fixing of components done by using nuts and bolts.

Figure 2 shows the $\mathrm{U}, \mathrm{L}$ and $\mathrm{C}$ shapes sheets provided by the versa robotics. Our vehicle consists of two main parts one is crawler body and the second one is the three degrees of freedom (3-DOF) manipulator arm with the gripper. Crawler which is the main frame of the vehicle holds tracked wheels, in order to move on the coarse surfaces easily without any difficulty facing.

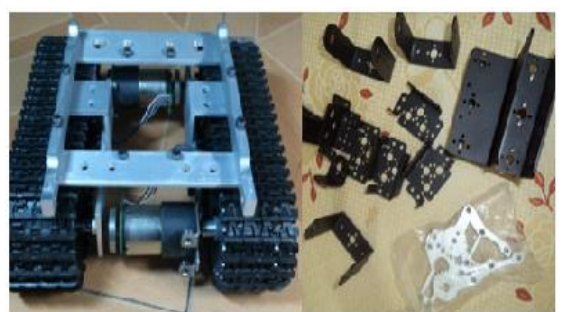

Fig. 2. Crawler robot and manipulator parts.

\subsection{Dc Geared Motor}

Source of moving the vehicle is motor. Our UGV consists of two de gear motors, track wheel drive based robot. Dc geared motors are used because they have low speed but high torque [8]. Always DC geared motor is used in that type of crawlers or tanks because at hilly or inclined surfaces more torque is needed instead of speed. Crawlers motor cannot allow the direct drive to the tracked- wheels because these motor's shaft is a lock or not compatible. In this type of robot special type of wheels are used named as spikey-wheels [9]. Table 1 shows the direct current (DC) motor specification of motors. 
TABLE I. DC Motor Specifications.

\begin{tabular}{|c|c|}
\hline Specification & Explanation \\
\hline Voltage Rated & $12 \mathrm{~V}$ \\
\hline Stall Current & $5.5 \mathrm{~A}$ \\
\hline No load current & $0.16 \mathrm{~A}$ \\
\hline Torque Rated & $0.53 \mathrm{Nm}$ \\
\hline Speed Rated & $327 \mathrm{rpm}$ \\
\hline Current Rated & $1.5 \mathrm{~A}$ \\
\hline Stall Torque & $2.15 \mathrm{Nm}$ \\
\hline
\end{tabular}

\subsection{Manipulator Arm}

Manipulator's assembly is made of aluminum $\mathrm{U}$ and $\mathrm{L}$ shapes plates. Purpose of placing manipulator in the robot is to pick and place the objects from the surface or ground [10].

This manipulator consists of shoulder, wrist, and gripper. Shoulder joint which is revolute and rotates about 90 degrees, wrist joint which is twisted joint rotate about 180 degree and last one is gripper whose angle of opening and closing is about 160 degree. Joints of manipulator robot are driven by 3 MG 996 Servo motors. There are 3 links in this manipulator robot each link is connected with the joint. Measurements of links are given below.

Distance between link 0 to link $1=96 \mathrm{~mm}$ (3.7 inch) this part is called shoulder.

Distance between links 1 to link $2=$ $105 \mathrm{~mm}$ (4.1 inches) this part is called wrist. With link 2 end effector is connected whose opening distance is about $55 \mathrm{~mm}$ ( $2.16 \mathrm{inch}$ ) Figure 3 shows the three degree of freedoms manipulator arm.

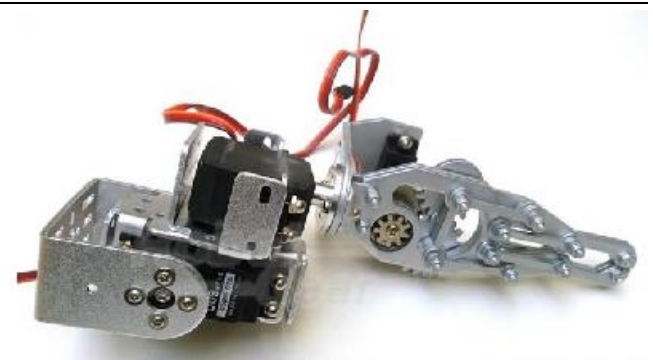

Fig. 3. 3-DOF manipulator robot arm.

Source: www.sainsmart.com/products/3axis-desktop-robotic-arm.

\subsection{Main Controller Arduino Uno}

This is the main controller of the vehicle. Consist of a microcontroller which saves the program which we write. This main board is operated at $5 \mathrm{~V} \mathrm{dc}$; we can supply it by 3 methods, through USB cable connected with PC, plug the 5V DC input at its VIN pin and connect the ground must and last one which is through external 5-12 Dc adapter to a power socket of the Arduino. You can give maximum supply $12 \mathrm{~V} \mathrm{dc}, 1 \mathrm{~A}$ current. Inside the Arduino, there is 7805 voltage regulator used which prevent the board from any damages. Figure 4 shows the input and output ports of the Arduino Uno. No need of soldering the wires user can easily plug the male connecting wires to the female header of Arduino UNO for communication.

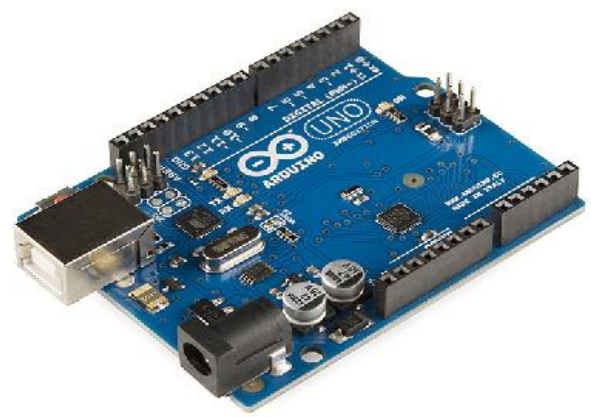

Fig. 4. Arduino Uno Main Controller. Source https://www.arduino.cc/ 


\subsection{Xbee Pro S1 Module}

This is a very interesting wireless device used for wireless communication between two points through buildings or any place. There are many Xbee modules such as Xbee S1 and Xbee S2, we are using Xbee Pro S1 this is the advanced version of the remaining XBee modules. Figure 5 shows the front view of xbee pro S1. This device is the product of Digi Tech [11]. It has a built-in antenna which makes the better communication, increase the distance of communication. These modules allow a very reliable and simple communication between microcontrollers, computers, systems, really anything with a serial port! Point to point and multi-point networks are supported with a range of 1500 meter coverage.

The Xbee Pro S1 module in this vehicle is used to provide the wireless communication between the unmanned ground vehicle and the surface controller.

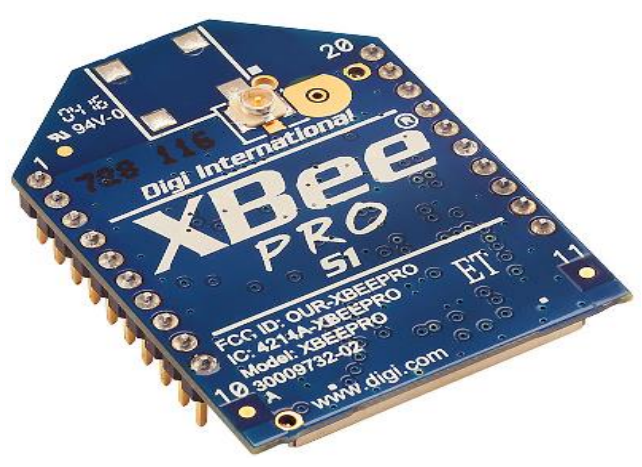

Fig. 5. Xbee Pro module. Source. https://www.digi.com

\subsection{Xbee X-CTU}

$\mathrm{X}$-CTU is software used to configure a connection between pc and xbee module. First we have to make the connection between pc and device. After this we select COM port where xbee is connected with the laptop port. After this we have to select correct baud rate which you set in the arduino, matching of baud rate is very important if there is no matching wireless system can't work. Figure 6 shows the interfacing window of the X-CTU.

From this software xbee input and output ports are initialized. After declaring of the ports proper selecting of sample rate is important in our case 20 is set.

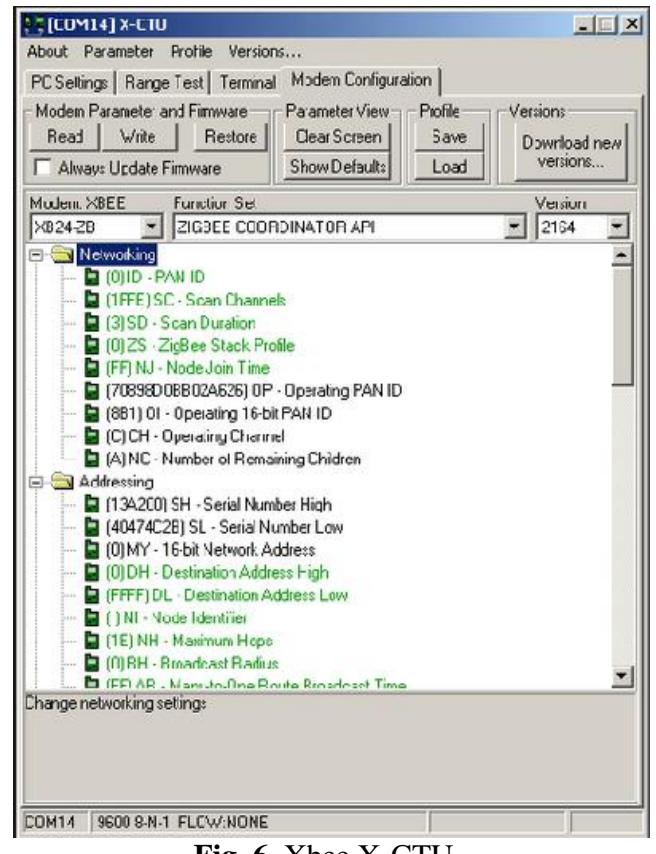

Fig. 6. Xbee X-CTU.

Source. https://www.digi.com

\section{Controlling The Robot Movement}

A controller is made for the controlling of UGV movements and manipulation of arm and camera, in which different switches and knobs are used. When user from the controller send the command through buttons or knobs, signal will be generated in the xbee and xbee sends the signal to the controller where signal is processing, then these signals transmitted to the UGV to perform the desired tasks. Figure 7 shows the controller of the robot., Table 1 show the input commands which sense the robot for specific movement and table 2 shows the main specifications of the UGV. 


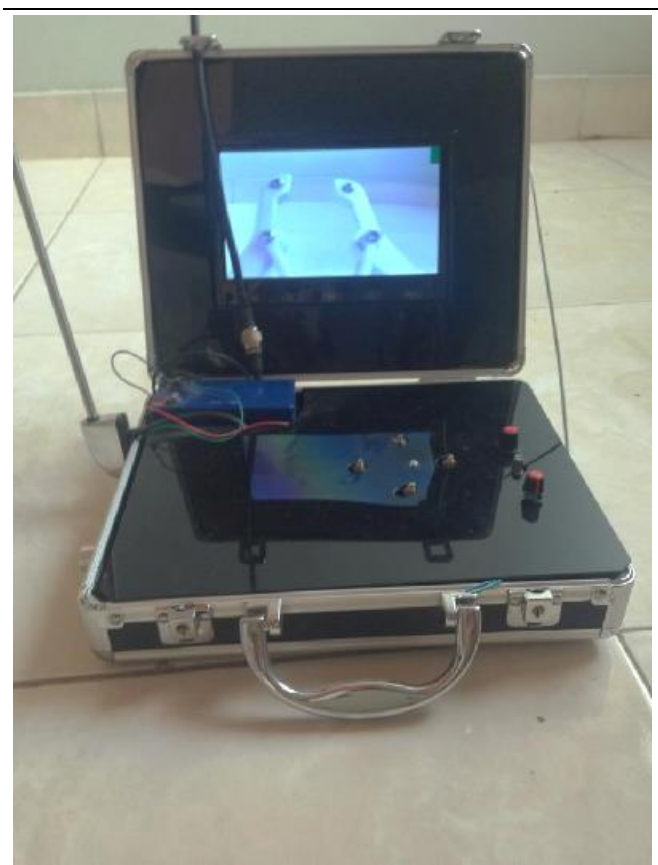

Fig. 7. Main controller of the robot.

TABLE II. Robot Commands.

\begin{tabular}{|l|l|}
\hline Input character & Command \\
\hline F & Move forward \\
\hline B & Move backward \\
\hline L & Turn left \\
\hline R & Turn right \\
\hline K1-Gripper & Open or Close \\
\hline K2- Shoulder & Lower or upper \\
\hline K3- Wrist & $\begin{array}{l}\text { Twist clock wise or } \\
\text { anti clockwise }\end{array}$ \\
\hline
\end{tabular}

TABLE III. UGV Specifications.

\begin{tabular}{|c|c|}
\hline Specification & Explanation \\
\hline UGV size ( L x W x H) & $\begin{array}{c}170 \times 167 \times 80 \\
\mathrm{~mm}\end{array}$ \\
\hline Crawler treads (width) & $50 \mathrm{~mm}$ \\
\hline Manipulator arm & $200 \mathrm{~mm}$ \\
\hline Controlling range & $\begin{array}{c}1000 \text { meter (open } \\
\text { area) }\end{array}$ \\
\hline Weight & $2 \mathrm{~kg}$ \\
\hline
\end{tabular}

Sukkur IBA Journal of Emerging Technologies - SJET $\mid$ Volu

\section{Results}

The execution of the unmanned ground vehicle (UGV) for search and rescue mission hardware model experimented throughout the real-time experiment. Figure 8 shows the experimental results of the unmanned ground vehicle with no load and full load torque, current and speed. It was affirmed that the model was flawlessly towing on both glossy and irregular surfaces. The dynamic strength of the crawling vehicle while transporting the objects was kept. The vehicle can operate remotely via Xbee from up to the boundary of 1000 meters coverage. The manipulator is proficient of grasping objects up to 1000 meters radius with live video feedback. The battery can serve for 8 hours outwardly renewing. Figure 9 shows the main model of the UGV.
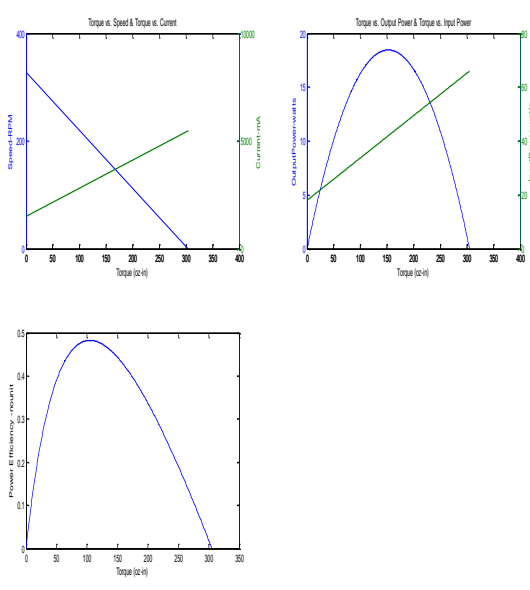

Fig. 8. UGV Load and No load results.

These figures are formulated in MATLAB version R2014b. Figure 8 shows the no load and full load speed, current, and torque of the unmanned ground vehicle on the ground. Therefore from figure we can see that slope of Torque Vs Current is 13.114754. The reciprocal is 0.076250 . Maximum output mechanical power is 18.450975(watts). This happens at the 
Torque load of 152.5 (oz-in), with Current $3500(\mathrm{~mA})$.

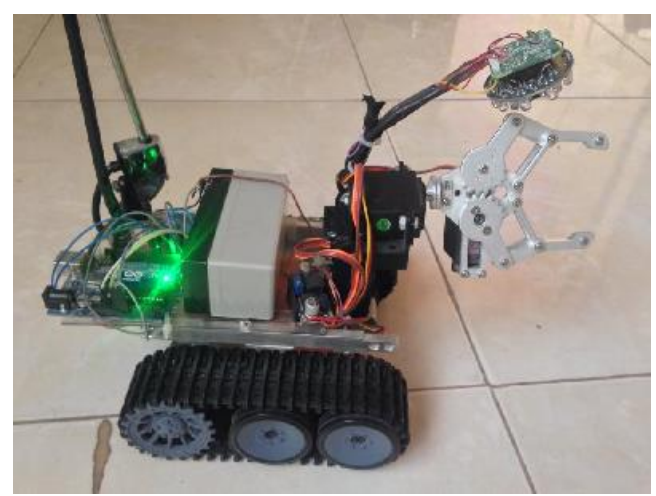

Fig. 9. Main model of the robot.

\section{Conslusion}

This article confers a scientific overview of the implementation of an unmanned ground vehicle with the three degree of freedom (3DOF) manipulator arm for search and rescue missions. A model was created for an apparent induction of the method and it has been decided that by this sort of system invented, search and rescue operations can be done with less human through appearance while rescuing, through keeping the human from dangers. The design method advised in this article is cost-effectively applying a fullduplex transmission and suitable for military search and monitoring missions.

\section{REFERENCES}

[1] J. Pyo, "Future Unmanned System Design, for Reliable Military Operations," International Journal of Control and Automation. Korea, vol. 5, pp. 173-186, September 2012.

[2] A. Bouhraoua, N. Merah, M. AlDajani and M. ElShafei, " Desing and Implementation of an Unmanned Geound Vehicle fo Security Applications", Proceedings of the 7th International Symposium on Mechatronics and its Applications. Saudi Arabia, vol. 4, pp. 20-22, April 2010
[3] D. Voth, "A New Generation of Military Robots", IEEE Intelligent Systems. USA, vol. 19, pp. 2-3, Jul-Aug 2004.

[4] S. Tsugaw, "Vision-based Vehicles in Japan: machine vision system and driving control systems", IEEE Transactions on Industrial Electronics. Vol. 41, pp. 398405, 1994.

[5] A. Bugaje, A. Loko, A. Ismail, A. Samuel, "The Unmanned Ground Vehicle for fumigation purpose". International Journal of Engineering Trends and Technology. Nigeria, vol 30, December 2015.

[6] S. Singh and P. Ranjan, "Towards a new low cost, simple implementation using embedded system wireless networking for UAVs", Proceedings of the IEEE 5th International Conference on Advanced Networks and Telecommunication Systems. India, pp. 18-21, December 2011.

[7] T.Gillespie,"Fundamentals of Vehicle Dynamics" SAE Interational, 1992.

[8] Y.Ali, S. Noor, and S. Bashi, "Microncontroller Performance for Dc Motor Speed Control System", Proceedings of Power Engineering Conference.Malaysia, December 2003.

[9] A. Mohebbi, S. Safaee, M. Keshmiri and S. Mohebbi, "Design, Simulation and Manufacturing of a Tracked Surveillance Unmanned Ground Vehicle", Proceedings of the IEEE International Conference on Robotics and Biomimetices. China, December 2010.

[10] B. Binoy, T. Keerthana, P. Barani, S.Aswathy, "A GSM based Versatile Unmanned Ground Vehicle", Proceedings of International Conference on Emerging Trends in Robotics and Communication Technologies. India, December 2010.

[11] D. Vidhya, N. Mapari, “ Wireless Control and Transmission of Data for Underwater Robot", International Journal for Innovative Research in Science and Technology. India, vol. 2, pp. 364-369, May 2016. 